\title{
A simple method to co-purify genomic DNA, RNA, and proteins for functional studies
}

Jian Jiang ${ }^{1}$, Junfei Ma ${ }^{1}$, Bin Liu, ${ }^{1, *}$ and Ying Wang1,,

${ }^{1}$ Department of Biological Sciences, Mississippi State University, Mississippi State, Mississippi 39762, USA

*Correspondence: binliu@biology.msstate.edu and wang@biology.msstate.edu

Keywords: co-purification; DNA, RNA, and proteins; protoplasts; viroid 


\section{Abstract}

Understanding the regulation of gene expression, from the epigenetic modifications on genomes to posttranscriptional and translational controls, are critical for elucidating molecular mechanisms underlying distinct phenotypes in biology. With the rapid development of Multi-Omics analyses, it is desirable to minimize sample variations by using DNA, RNA, and proteins co-purified from the same samples. Currently, most of the co-purification protocols rely on Tri Reagent (Trizol as a common representative) and require protein precipitation and dissolving steps, which render difficulties in experimental handling and high-throughput analyses. Here, we established a simple and robust method to minimize the precipitation steps and yield ready-to-use RNA and protein in solutions. This method can be applied to samples in small quantity, such as protoplasts. We demonstrated that the protoplast system equipped with this method may facilitate studies on viroid biogenesis. Given the ease and the robustness of this new method, it will have broad applications for plant research and other disciplines in molecular biology.

\section{Introduction}

The flow of genetic information from genome to transcriptome and then proteins dictates the phenotype in all organisms (Crick, 1970). The genomic cis-regulatory elements and epigenetic hallmarks (e.g., cytosine methylation) in genomic DNA sequences may help elucidate transcriptional regulations of genes expression, the effects of which may impact the corresponding mRNA and protein levels (Hollick, 2017, Cheng et al., 2018, Zhang et al., 2018). Comparison between mRNA and protein expression profiles can help uncover possible post-transcriptional (Cheng and Chen, 2004, Liu and Chen, 2016, Kawa and 
Testerink, 2017) and/or translational controls (Skelly et al., 2016, Sablok et al., 2017). Furthermore, the function of a given gene can be inferred by its temporal-spatial patterns associated with a given phenotype. When equipped with various high-throughput technologies, such analyses can be expanded to the whole genomic level (Brautigam and Gowik, 2010, Reinders and Paszkowski, 2010, Jackson et al., 2011, Matros et al., 2011, Schmitz and Zhang, 2011, Schneeberger and Weigel, 2011, Huang and Han, 2014). With the development of numerous bioinformatics tools, many studies have begun to employ Multi-Omics approaches in order to elucidate regulatory networks governing gene expression that consequently regulates diverse phenotypes or biological processes (Zheng et al., 2017, Choi, 2018, Liu et al., 2018, Shang and Huang, 2018).

It is notable that many genes exhibit rapid and sensitive responses to the surrounding environment, which makes collecting experimental materials from the same sample a necessity to ensure the maximum accuracy for subsequent analyses. Simultaneously extracting DNA, RNA, and proteins is possible through a guanidine isothiocyanate-based protocol (Coombs et al., 1990), and several derivatives using Tri Reagent (containing guanidine isothiocyanate as a major component) have been developed with minor modifications (Tolosa et al., 2007, Radpour et al., 2009, Xiong et al., 2011, Rajput et al., 2012, Pena-Llopis and Brugarolas, 2013, Vorreiter et al., 2016). Generally, Tri Reagent separates DNA, RNA, and proteins to different fractions. Sequential precipitation or its combination with specific nucleic acid binding columns facilitate further purification. However, almost all those protocols require protein precipitation and dissolving steps, increasing difficulties in experimental handling and reducing yields. In addition, those 
protocols may not be as effective for valuable samples in small quantity, which further restrains their applications in research.

Here, we established a simple and robust method to co-purify DNA, RNA, and proteins from the same sample. This method does not employ Tri Reagent, has minimum precipitation steps, and generates ready-to-use RNA and protein solutions with high quality. Our analysis showed that both nuclear and cytosolic proteins can be effectively purified. Of note, this method is compatible with samples in small quantity.

We attempted to employ the powerful Arabidopsis genetic resources to facilitate studies on viroid biogenesis, using the protoplast replication system. Viroids are circular non-coding RNAs that mainly infect crop plants (Ding, 2009, Flores et al., 2014, Flores et al., 2016). The host machinery and the underlying mechanisms for viroid biogenesis are largely unclear due to technical challenges in biochemical approaches. Viroids cannot embark systemic infection in $A$. thaliana (Ding, 2009), which hindered the usage of Arabidopsis genetic resources for viroid research. However, multiple viroids can replicate in Arabidopsis transgenic lines expressing their cDNAs, indicating the presence of conserved machinery for viroid biogenesis (Daros and Flores, 2004). Here, we demonstrated the replication of potato spindle tuber viroid (PSTVd) in Arabidopsis protoplasts and successfully applied our method to co-purify DNA, RNA, and proteins. This experimental platform will significantly enhance our capacity to probe the host machinery and the functional mechanisms for PSTVd nuclear import/export and propagation. Moreover, our new method should have broad applications for various research in plant biology and other disciplines in molecular biology. 


\section{Results}

\section{Recovering proteins after RNA enrichment}

We chose transgenic Nicotiana benthamiana 16C plant with constitutive GFP expression as the test materials and used GeneJET plant RNA purification kit and MagJET RNA purification kit for RNA purification. The total RNA was purified following manufacturer's instructions. It is notable that the MagJET family has a variety of products to purify different RNA species, which is ideal for one-step enrichments of desired RNA populations for downstream analyses. As expected, we could easily detect GFP mRNA using total RNA purified from either the GeneJET plant RNA purification kit or the MagJET RNA kit by Reverse Transcription (RT)-PCR (Figure 1).

Because proteins normally pass through the column or remain in the supernatant after RNA binding to the magnetic beads, we reasoned that it is possible to recover proteins from the flow-through fraction (FL) after RNA binding to the GeneJET column or the unbound supernatant fraction (Su) after RNA binding to the MagJET beads. Interestingly, the SDS-PAGE Sample Prep Kit employs DMSO-denaturing-based principle to enrich proteins and remove undesired chemicals from protein solutions. Therefore, we decided to test whether a combinational usage of both RNA and protein purification kits can purify RNA, and proteins from the same sample. We added DMSO to the FL and Su fractions and applied the mixtures to the SDS-PAGE Sample Prep Kit to enrich total proteins. After applying the enriched proteins to SDS-PAGE gel electrophoresis and silver staining, we observed effective recovery of total proteins from both the FL and Su fractions. When comparing the recovering efficiency with proteins directly purified from leaf samples using RIPA buffer, recovery from FL and Su are less than the direct RIPA purification (Figure 
2). Nevertheless, most of the protein bands in RIPA buffer purified samples are present in samples prepared with our method. Furthermore, the consistent protein patterns in the replicates using our method indicates that this new method is reliable for protein analyses. As a further test, we performed an immunoblotting assay and detected the presence of GFP protein in recovered proteins using our purification method (Figure 3). Interestingly, the GFP signals were stronger in protein recovered from the FL fraction as compared with proteins directly purified using RIPA buffer, likely due to the removal of undesired chemicals by the SDS-PAGE Sample Prep Kit. Since the Su fraction already contains a high concentration of ethanol that can denature proteins, we tested if proteins can be effectively recovered using the SDS-PAGE Sample Prep Kit without supplementing DMSO. As shown in Figure 4, silver staining of total proteins and immunoblotting detection of GFP demonstrated that omitting DMSO slightly reduced the protein recovery yield but still provided a desired recovery of proteins. Since supplementing DMSO to the RNA-depleted solution significantly increases the solution volume and centrifugation steps, it is possible to omit DMSO to improve the speed of purification process for highthroughput analyses.

\section{DNA recovery from tissue debris using DNAzol-ES}

Some studies require analysis of genomic DNA. Therefore, it is desirable to purify genomic DNA from the same sample together with the purification of RNA, and proteins. We reasoned that the tissue debris as the leftover from RNA purification steps contains a sufficient amount of genomic DNA that can be purified and analyzed. We applied DNAzol-ES to the debris and followed the purification protocol from the manufacturer. 
From six $N$. benthamiana seedlings $(<1 \mathrm{mg})$, we recovered genomic DNA from the debris (157.5 $\mathrm{ng}$ and $192.5 \mathrm{ng}$ in two replicates). The recovery rate is about 5 - to 7 -fold less as compared with the direct DNAzol-ES purification (850 ng and $1090 \mathrm{ng}$ in two replicates) using the same amount of starting materials. Nonetheless, it still provides a reasonable amount of genomic DNA for subsequent analyses. PCR amplification of a GFP fragment showed that the genomic DNA purified from the debris are suitable for downstream molecular analysis. It is notable that if only genomic DNA and RNA are desired for analyses, a combinational use of RNAzol and DNAzol-ES as instructed in manuals will maximize the recovery of genomic DNA and RNA.

With the successful recovery of genomic DNA from the same sample, we established an effective method to co-purify genomic DNA, RNA, and proteins from the same sample. As illustrated in Figure 5, samples are subjected to RNA purification by various commercial kits, and the flow-through fraction or the unbound supernatant are used for protein recovery using the SDS-PAGE Sample Prep Kit. Genomic DNA is recovered from tissue debris using DNAzol-ES.

\section{Co-purification of DNA, RNA, and proteins from protoplasts}

We are interested in testing whether this method is efficient for samples in small quantity, such as protoplasts. Protoplast system allows rapid analysis of protein subcellular localization (Priyadarshani et al., 2018, Rolland, 2018), gene functions (Hamel et al., 2011, Li et al., 2013), plant responses to stresses (Asai et al., 2002, He et al., 2007), viral replication processes (Qi and Ding, 2002, Owen et al., 2016), etc. However, previous studies were restrained to microscopic analysis or to only analyze RNA or proteins due 
to the limited amount of materials. Here, we used PSTVd to co-transfect $A$. thaliana protoplasts with GFP reporter plasmids (35S::GFP). PSTVd replicates in $A$. thaliana (Daros and Flores, 2004, Itaya et al., 2007) but cannot achieve systemic trafficking (Daros and Flores, 2004). In our test, about $2 \times 10^{5}$ protoplast cells were harvested two days posttransfection and were subjected to nucleic acids and protein purification. As shown in Figure 6a, we could detect PSTVd replication using RNA gel blots, as indicated by the presence of circular genomic RNA. Immunoblots detected the expression of GFP, with Histone H3 as a loading control (Figure 6b). Genomic PCR was successfully performed to amplify a fragment of the endogenous Transcription Factor IIIA (TFIIIA) gene (Figure $6 c)$. These results demonstrated that this new method is efficient for materials in small quantity. Importantly, these results also showed that our new method can effectively recover proteins from both cytosolic and nuclear compartments.

\section{Discussion}

With the rapid development of various Omics technologies, there has been a trend to analyze Multi-Omics data coordinately to achieve a better understanding of the complex gene networks in various biological processes (Brautigam and Gowik, 2010). Thus, it is critical to fraction and purify distinct biological components (e.g., DNA, RNA, and protein) with high quality for analyses. It is notable that many of the genes exhibit rapid expression dynamics in response to diverse environmental stimuli, so using distinct components from the same sample will overtly enhance the accuracy of the subsequent analyses. In this regard, methods to co-purify various biological components from the same sample are necessities. Currently, most of the available protocols to co-purify DNA, RNA, and 
proteins from the same sample are largely based on Tri Reagent and involve multiple precipitation steps (Vorreiter et al., 2016). These protocols all require precipitation of proteins and then dissolving the pellets, which are time-consuming and technically challenging. Here, by using the SDS-PAGE sample prep kit, we developed a method that can purify proteins after RNA purification using various kits (column-based or magnetic bead-based). The genomic DNA can then be purified from tissue debris that is the leftover from the initial RNA purification steps. Of note, RNA was purified in the first step, which ensures the yield of high-quality RNA with minimum degradation while waiting. The robustness of the SDS-PAGE Sample Prep Kit in total protein recovery makes the method potentially compatible with a wide range of RNA purification products. This method is simple and straightforward and generates ready-to-use materials for downstream immunoblotting analysis, RNA gel electrophoresis analyses, regular cloning, or Nextgeneration Deep Sequencing analyses.

One advantage of our method is that it is compatible with samples in small quantities as starting materials, such as protoplasts. Protoplast assays have been widely used in plant biology to rapidly and efficiently test: 1) the cellular localization of proteins and RNAs (Priyadarshani et al., 2018, Rolland, 2018), 2) plant responses to biotic stresses at transcriptional and post-transcriptional levels (Asai et al., 2002, He et al., 2007), 3) protein-protein interactions (Schweiger and Schwenkert, 2014, Priyadarshani et al., 2018), 4) gene functions (Hamel et al., 2011, Li et al., 2013), 5) viral replications (Qi and Ding, 2002, Owen et al., 2016), etc. Due to the limit of sample quantity, current studies are often limited to microscopic analysis or only analyze one type of biological components (DNA, 
RNA, or protein) in protoplasts, undermining the value of this transient transgenic approach.

Here, we demonstrated that the combination of the Arabidopsis protoplast replication system and the co-purification system can significantly advance viroid research by opening the door to the powerful Arabidopsis genetic resources (Li et al., 2014). Viroids are circular noncoding RNAs that infect crop plants, often leading to plant disease (Ding, 2009, Flores et al., 2014). As infectious noncoding RNAs, viroids have been a productive model to dissect plant defense mechanisms against invasive RNAs (Itaya et al., 2007, StPierre et al., 2009, Zheng et al., 2017), the role of RNA three-dimensional motifs in regulating RNA systemic trafficking (Ding, 2009, Wang et al., 2018), and RNA-templated RNA replication by DNA-dependent RNA polymerase II (Pol II) (Rackwitz et al., 1981, Wang et al., 2016). Recent progress has begun to elucidate how viroids co-opt cellular factors to effectively propagate themselves (Flores et al., 2005, Ding, 2009, Nohales et al., 2012a, Nohales et al., 2012b, Minoia et al., 2014, Katsarou et al., 2016, Wang et al., 2016, Dissanayaka Mudiyanselage et al., 2018, Jiang et al., 2018). PSTVd, and viroids in family Pospiviroidae, employ Pol II for transcription (Rackwitz et al., 1981). A splicing variant of TFIIIA (TFIIIA-7ZF) facilitates Pol II-catalyzed transcription using PSTVd RNA template (Wang et al., 2016). Interestingly, the splicing of TFIIIA transcripts is regulated through a direct interaction between PSTVd and ribosomal protein L5 (RPL5), which results in optimal expression of TFIIIA-7ZF favoring PSTVd transcription (Jiang et al., 2018). During replication, the ligation of linear unit-length of PSTVd is catalyzed by host DNA ligase 1 (Nohales et al., 2012a). It is interesting that a PSTVd relative, hop stunt viroid, can directly interact with host Histone deacetylase 6 and alter the DNA methylation 
patterns in host genome (Castellano et al., 2016). Viroids in family Avsunviroidae utilize

a nuclear-encoded chloroplastic DNA-dependent RNA polymerase for transcription

(Navarro et al., 2000). During processing, a chloroplastic RNA binding protein PARBP33, binds Avocado sunblotch viroid (ASBVd) and facilitates ASBVd self-cleavage (Daros and Flores, 2002). The monomeric linear ASBVd RNA is circularized by a chloroplastic isoform of tRNA ligase (Nohales et al., 2012b). In spite of these progresses, future studies are required to unravel other cellular factors involved in viroid biogenesis and their functional mechanisms. Using the Arabidopsis protoplast system in combination with our co-purification method, viroid research can now take advantage of the potent genetic resource for future explorations. Our method present here is also applicable to various other research in molecular biology.

\section{Experimental procedures}

\section{Plant growth and protoplast}

$N$. benthamiana plants were grown in a growth chamber at $25^{\circ} \mathrm{C}$ and with a $16 / 8 \mathrm{hr}$ light/dark cycle. $A$. thaliana plants were grown in a growth chamber at $23^{\circ} \mathrm{C}$ and with a 16/8 hr light/dark cycle. Using 4-week old Arabidopsis plants, protoplasts were isolated following a published protocol (Wu et al., 2009). Briefly, we used 3\% cellulose (Onozuka Yakult Pharmaceutical IND., Tokyo, Japan) and $0.8 \%$ macerase (MilliporeSigma, Burlington, MA) in digestion buffer (0.4 M mannitol, $20 \mathrm{mM} \mathrm{KCl,} 20 \mathrm{mM}$ MES, $10 \mathrm{mM}$ $\mathrm{CaCl}_{2}, 0.1 \% \mathrm{BSA}, 5 \mathrm{mM} \beta$-mercaptoethanol, $\left.\mathrm{pH} 5.7\right)$ to digest leaves with the lower epidermis layer removed by tapes. After $1 \mathrm{hr}$ digestion, the protoplasts were pelleted using $1 \mathrm{~min}$ centrifugation at $150 \mathrm{~g}$. The pelleted protoplasts were then sequentially 
incubated in the W5 buffer (5 mM MES, $154 \mathrm{mM} \mathrm{NaCl}, 125 \mathrm{mM} \mathrm{CaCl}, 5 \mathrm{mM} \mathrm{KCl}, \mathrm{pH}$ 5.7) on ice and MMg solution (4 mM MES, 0.4 M mannitol, $15 \mathrm{mM} \mathrm{MgCl}_{2}, \mathrm{pH}$ 5.7) at room temperature. About $10^{5}$ cells in $200 \mu \mathrm{MMg}$ buffer were supplemented with $40 \mu \mathrm{g}$ 35S::GFP plasmid and $5 \mu \mathrm{g}$ RZ-PSTVd ${ }^{\text {Int }}$ RNA (Itaya et al., 2007), then mixed with 200

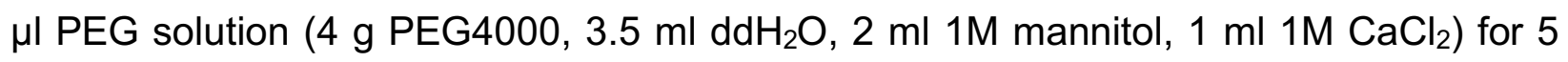
min incubation. Finally, protoplasts were washed with W5 solution and then incubated in WI solution (0.4 M mannitol, $20 \mathrm{mM} \mathrm{KCl,} 20 \mathrm{mM}$ MES, pH 5.7) for 2 days before RNA purification.

\section{RNA extraction and analysis}

Leaf samples were collected in $1.5 \mathrm{ml}$ microcentrifuge tubes and ground in liquid nitrogen. The GeneJET plant RNA purification kit and the MagJET RNA Kit (Thermo Fisher Scientific, Waltham, MA) were used for RNA purification as instructed in the manuals. Briefly, when using the GeneJET plant RNA purification kit, tissue lysate was loaded into the column for centrifugation. The flow-through (FL) was collected for subsequent protein purification. The column was washed twice using washing buffer from the kit. Nucleasefree water was used to elute the total RNA from the column after the DNase I treatment. When using the MagJET kit, $40 \mu$ of MagJET magnetic beads and $400 \mu$ l of ethanol (96$100 \%$ ) were added to $400 \mu$ tissue lysate free of tissue debris, and the mixture was kept rotating for $5 \mathrm{~min}$ in room temperature. The mixture was then placed in the magnetic rack for $1 \mathrm{~min}$. The unbound supernatant fractions (Su) were collected for protein purification. The magnetic beads were subjected to DNase I treatment and sequential washes for three times. Finally, $100 \mu$ l of nuclease-free water was added to beads to elute total RNA. 
The flow-through solution after RNA binding to GeneJET column and the supernatant solution collected after RNA binding to the MagJET beads were kept on ice for protein purification. The pellets tissue debris from both purification kits were saved for genomic DNA extraction. For RNA purification from protoplasts, the cells were pelleted through $1,000 \mathrm{~g}$ centrifugation for $2 \mathrm{~min}$ and then directly subjected to RNA purification using the MagJET RNA kit.

\section{RNA analysis using RT-PCR and RNA gel blots}

For RNA analysis, total RNA extracted from above methods was subjected to reverse transcription (RT) using Superscript III reverse transcriptase (Thermo Fisher Scientific). We followed the manufacturer manual for first strand synthesis. The first strand product was subjected to PCR using primers specific for GFP mRNA (16C f: 5'ctccacaacgtatacatcatggc-3' and 16C r: 5'-ccatgccatgtgtaatcccagcag-3'). For RNA gel blotting, we followed the protocol described previously. Briefly, total RNA was electrophoresed on $5 \%(\mathrm{w} / \mathrm{v})$ polyacrylamide/8M urea gels for $1 \mathrm{hr}$ at $200 \mathrm{~V}$, transferred to Hybond-XL nylon membranes (GE Healthcare Life Sciences, Pittsburgh, PA) using a semi-dry transfer unit (Bio-Rad, Hercules, CA), and immobilized by UV cross-linking. The membrane was blocked using Denhardt solution (VWR, Radnor, PA) followed by overnight hybridization with DIG-labeled riboprobe at $65^{\circ} \mathrm{C}$. DIG-labeled PSTVd specific probe was generated using Hindll-linearized plnt(-) plasmid (Qi and Ding, 2002) and a T7 polymerase MAXIscript kit (Thermo Fisher Scientific). Following the instructions of a DIG northern starter kit (MilliporeSigma), the membranes were washed and incubated with the antibody against DIG labeling. The alkaline phosphatase substrates were applied to the 
membranes, followed by fluorescence signal detection using C-Digit (Li-COR Biosciences, Lincoln, NE).

\section{Protein extraction}

The FL fraction from the GeneJET kit and the Su fraction from the MagJET kit were subjected for protein purification, using the Pierce SDS-PAGE Sample Prep Kit (Thermo Fisher Scientific). Briefly, the liquid fractions supplemented with DMSO were directly loaded into a spin column. The samples were subjected to centrifugation at 2,000 g for 2 min. The flow-through was discarded. Columns were washed twice using the wash buffer and incubated with the elution buffer at $60^{\circ} \mathrm{C}$ for $5 \mathrm{~min}$, followed by centrifugation at 2,000 $\mathrm{g}$ for 2 min to collect protein solutions. For RIPA buffer purification, leaf powders were mixed with 1X RIPA directly and the supernatants were used for analyses.

\section{Silver staining and Immunoblotting}

The purified proteins were subjected to SDS-PAGE separation and transferred to nitrocellulose membranes using a semidry transfer unit (Bio-Rad). After 10 min incubation with Rapidblock solution (VWR), primary antibodies against GFP or Histone $\mathrm{H} 3$ (Genscript, Piscataway, NJ) were used at 1:2,000 dilution for overnight incubation at $4^{\circ} \mathrm{C}$. After three washes with 1X TBST, HRP-conjugated secondary antibody against rabbit (MilliporeSigma) was added at 1:3,000 dilution for detecting Histone $\mathrm{H} 3$, while HRPconjugated secondary antibody against mouse (MilliporeSigma) was added at 1:8,000 dilution for detecting GFP. After 1X TBST wash for three times and then incubation with HRP substrates (Li-COR Biosciences), the signals were captured with C-Digit (Li-COR 
Biosciences). For silver staining, the gels after electrophoresis were treated with the Silver Bullit $^{\mathrm{TM}}$ kit (VWR), following instructions in the user manual.

\section{DNA extraction}

The pellet of cell debris left from RNA purification was subject to $1 \mathrm{ml}$ DNAzol-ES following instructions from the vendor (Molecular research center Inc., Cincinnati, OH). The mixture was subject to centrifugation at $10,000 \mathrm{~g}$ for $10 \mathrm{~min}$, and the supernatant was then transferred to a new centrifuge tube. Then $500 \mu \mathrm{l} 100 \%$ ethanol was added to the supernatant and mixed at room temperature for $3 \mathrm{~min}$. Genomic DNA was precipitated at $5,000 \mathrm{~g}$ centrifugation for $5 \mathrm{~min}$. The DNA pellet was washed twice with $1 \mathrm{ml}$ ice cold $75 \%$ ethanol and air dried for $5 \mathrm{~min}$. The pellet was dissolved using $20 \mu \mathrm{l} \mathrm{TE}$ buffer. The purified genomic DNA samples, after dilution to equal concentration $(\sim 10 \mathrm{ng} / \mu \mathrm{l})$, were subjected to PCR analysis to detect GFP gene in 16C plants (primers described above) or TFIIIA fragment in Arabidopsis (AtTFIIIA genome p f: 5'-ggagacctcctgagaagctccagc-3' and AtTFIIIA genome p r: 5'-gtccttatcacggttgtcattactatg-3'). PCR product was confirmed using agarose gel electrophoresis.

\section{Acknowledgements}

We are grateful for David Baulcombe at University of Cambridge for sharing the $N$. benthamiana 16C line as a gift. We are thankful for Donna Gordon at Mississippi State University for constructive discussions. We thank Shachinthaka Dissanayaka Mudiyanselage at Mississippi State University for critical reading. This work was supported by US National Science Foundation (IOS-1564366) to YW; The Strategic 
Research Initiative fund from College of Arts and Sciences to YW; and American Heart Association under grant to $\mathrm{BL}$.

\section{Conflicts of interest}

No potential conflict of interest was disclosed.

\section{References}

Asai, T., Tena, G., Plotnikova, J., Willmann, M.R., Chiu, W.L., Gomez-Gomez, L., Boller, T., Ausubel, F.M. and Sheen, J. (2002) MAP kinase signalling cascade in Arabidopsis innate immunity. Nature, 415, 977-983.

Brautigam, A. and Gowik, U. (2010) What can next generation sequencing do for you? Next generation sequencing as a valuable tool in plant research. Plant Biol (Stuttg), 12, 831-841.

Castellano, M., Pallas, V. and Gomez, G. (2016) A pathogenic long noncoding RNA redesigns the epigenetic landscape of the infected cells by subverting host Histone Deacetylase 6 activity. New Phytol, 211, 1311-1322.

Cheng, F., Wu, J., Cai, X., Liang, J., Freeling, M. and Wang, X. (2018) Gene retention, fractionation and subgenome differences in polyploid plants. Nat Plants, 4, 258-268.

Cheng, Y. and Chen, X. (2004) Posttranscriptional control of plant development. Curr Opin Plant Biol, 7, 20-25.

Choi, H.K. (2018) Translational genomics and multi-omics integrated approaches as a useful strategy for crop breeding. Genes Genomics.

Coombs, L.M., Pigott, D., Proctor, A., Eydmann, M., Denner, J. and Knowles, M.A. (1990) Simultaneous isolation of DNA, RNA, and antigenic protein exhibiting kinase activity from small tumor samples using guanidine isothiocyanate. Anal Biochem, 188, 338-343.

Crick, F. (1970) Central dogma of molecular biology. Nature, 227, 561-563.

Daros, J.A. and Flores, R. (2002) A chloroplast protein binds a viroid RNA in vivo and facilitates its hammerhead-mediated self-cleavage. EMBO J, 21, 749-759.

Daros, J.A. and Flores, R. (2004) Arabidopsis thaliana has the enzymatic machinery for replicating representative viroid species of the family Pospiviroidae. Proc Natl Acad Sci U $S$ A, 101, 6792-6797.

Ding, B. (2009) The biology of viroid-host interactions. Annu Rev Phytopathol, 47, 105-131.

Dissanayaka Mudiyanselage, S.D., Qu, J., Tian, N., Jiang, J. and Wang, Y. (2018) Potato Spindle Tuber Viroid RNA-Templated Transcription: Factors and Regulation. Viruses, 10.

Flores, R., Gago-Zachert, S., Serra, P., Sanjuan, R. and Elena, S.F. (2014) Viroids: survivors from the RNA world? Annu Rev Microbiol, 68, 395-414.

Flores, R., Hernandez, C., Martinez de Alba, A.E., Daros, J.A. and Di Serio, F . (2005) Viroids and viroid-host interactions. Annu Rev Phytopathol, 43, 117-139.

Flores, R., Owens, R.A. and Taylor, J. (2016) Pathogenesis by subviral agents: viroids and hepatitis delta virus. Curr Opin Virol, 17, 87-94. 
Hamel, L.P., Benchabane, M., Nicole, M.C., Major, I.T., Morency, M.J., Pelletier, G., Beaudoin, N., Sheen, J. and Seguin, A. (2011) Stress-responsive mitogen-activated protein kinases interact with the EAR motif of a poplar zinc finger protein and mediate its degradation through the $26 \mathrm{~S}$ proteasome. Plant Physiol, 157, 1379-1393.

He, P., Shan, L. and Sheen, J. (2007) The use of protoplasts to study innate immune responses. Methods Mol Biol, 354, 1-9.

Hollick, J.B. (2017) Paramutation and related phenomena in diverse species. Nat Rev Genet, 18, 5-23.

Huang, X. and Han, B. (2014) Natural variations and genome-wide association studies in crop plants. Annu Rev Plant Biol, 65, 531-551.

Itaya, A., Zhong, X., Bundschuh, R., Qi, Y., Wang, Y., Takeda, R., Harris, A.R., Molina, C., Nelson, R.S. and Ding, B. (2007) A structured viroid RNA serves as a substrate for dicerlike cleavage to produce biologically active small RNAs but is resistant to RNA-induced silencing complex-mediated degradation. J Virol, 81, 2980-2994.

Jackson, S.A., Iwata, A., Lee, S.H., Schmutz, J. and Shoemaker, R. (2011) Sequencing crop genomes: approaches and applications. New Phytol, 191, 915-925.

Jiang, J., Smith, H.N., Ren, D., Dissanayaka, S.M., Dawe, A.L., Wang, L. and Wang, Y. (2018) Potato spindle tuber viroid modulates its replication through a direct interaction with a splicing regulator. $J$ Virol.

Katsarou, K., Mavrothalassiti, E., Dermauw, W., Van Leeuwen, T. and Kalantidis, K. (2016) Combined Activity of DCL2 and DCL3 Is Crucial in the Defense against Potato Spindle Tuber Viroid. PLoS Pathog, 12, e1005936.

Kawa, D. and Testerink, C. (2017) Regulation of mRNA decay in plant responses to salt and osmotic stress. Cell Mol Life Sci, 74, 1165-1176.

Li, D., Dreher, K., Knee, E., Brkljacic, J., Grotewold, E., Berardini, T.Z., Lamesch, P., Garcia-Hernandez, M., Reiser, L. and Huala, E. (2014) Arabidopsis database and stock resources. Methods Mol Biol, 1062, 65-96.

Li, J.F., Chung, H.S., Niu, Y., Bush, J., McCormack, M. and Sheen, J. (2013) Comprehensive protein-based artificial microRNA screens for effective gene silencing in plants. Plant Cell, 25, 1507-1522.

Liu, F., Marshall, R.S. and Li, F. (2018) Understanding and exploiting the roles of autophagy in plants through multi-omics approaches. Plant Sci, 274, 146-152.

Liu, L. and Chen, X. (2016) RNA Quality Control as a Key to Suppressing RNA Silencing of Endogenous Genes in Plants. Mol Plant, 9, 826-836.

Matros, A., Kaspar, S., Witzel, K. and Mock, H.P. (2011) Recent progress in liquid chromatography-based separation and label-free quantitative plant proteomics. Phytochemistry, 72, 963-974.

Minoia, S., Carbonell, A., Di Serio, F., Gisel, A., Carrington, J.C., Navarro, B. and Flores, R. (2014) Specific argonautes selectively bind small RNAs derived from potato spindle tuber viroid and attenuate viroid accumulation in vivo. $J$ Virol, 88, 11933-11945.

Navarro, J.A., Vera, A. and Flores, R. (2000) A chloroplastic RNA polymerase resistant to tagetitoxin is involved in replication of avocado sunblotch viroid. Virology, 268, 218-225.

Nohales, M.A., Flores, R. and Daros, J.A. (2012a) Viroid RNA redirects host DNA ligase 1 to act as an RNA ligase. Proc Natl Acad Sci U S A, 109, 13805-13810. 
Nohales, M.A., Molina-Serrano, D., Flores, R. and Daros, J.A. (2012b) Involvement of the chloroplastic isoform of tRNA ligase in the replication of viroids belonging to the family Avsunviroidae. J Virol, 86, 8269-8276.

Owen, C.A., Moukarzel, R., Huang, X., Kassem, M.A., Eliasco, E., Aranda, M.A., Coutts, R.H. and Livieratos, I.C. (2016) In Vitro Synthesized RNA Generated from cDNA Clones of Both Genomic Components of Cucurbit yellow stunting disorder virus Replicates in Cucumber Protoplasts. Viruses, 8.

Pena-Llopis, S. and Brugarolas, J. (2013) Simultaneous isolation of high-quality DNA, RNA, miRNA, and proteins from tissues for genomic applications. Nat Protoc, 8, 2240-2255.

Priyadarshani, S., Hu, B., Li, W., Ali, H., Jia, H., Zhao, L., Ojolo, S.P., Azam, S.M., Xiong, J., Yan, M., Ur Rahman, Z., Wu, Q. and Qin, Y. (2018) Simple protoplast isolation system for gene expression and protein interaction studies in pineapple (Ananas comosus L.). Plant Methods, 14, 95.

Qi, Y. and Ding, B. (2002) Replication of Potato spindle tuber viroid in cultured cells of tobacco and Nicotiana benthamiana: the role of specific nucleotides in determining replication levels for host adaptation. Virology, 302, 445-456.

Rackwitz, H.R., Rohde, W. and Sanger, H.L. (1981) DNA-dependent RNA polymerase II of plant origin transcribes viroid RNA into full-length copies. Nature, 291, 297-301.

Radpour, R., Sikora, M., Grussenmeyer, T., Kohler, C., Barekati, Z., Holzgreve, W., Lefkovits, I. and Zhong, X.Y. (2009) Simultaneous isolation of DNA, RNA, and proteins for genetic, epigenetic, transcriptomic, and proteomic analysis. J Proteome Res, 8, 52645274.

Rajput, S.K., Dave, V.P., Rajput, A., Pandey, H.P., Datta, T.K. and Singh, R.K. (2012) A column-based rapid method for the simultaneous isolation of DNA, RNA, miRNA, and proteins. Cell Biol Int, 36, 779-783.

Reinders, J. and Paszkowski, J. (2010) Bisulfite methylation profiling of large genomes. Epigenomics, 2, 209-220.

Rolland, V. (2018) Determining the Subcellular Localization of Fluorescently Tagged Proteins Using Protoplasts Extracted from Transiently Transformed Nicotiana benthamiana Leaves. Methods Mol Biol, 1770, 263-283.

Sablok, G., Powell, J.J. and Kazan, K. (2017) Emerging Roles and Landscape of Translating mRNAs in Plants. Front Plant Sci, 8, 1443.

Schmitz, R.J. and Zhang, X. (2011) High-throughput approaches for plant epigenomic studies. Curr Opin Plant Biol, 14, 130-136.

Schneeberger, K. and Weigel, D. (2011) Fast-forward genetics enabled by new sequencing technologies. Trends Plant Sci, 16, 282-288.

Schweiger, R. and Schwenkert, S. (2014) Protein-protein interactions visualized by bimolecular fluorescence complementation in tobacco protoplasts and leaves. $J$ Vis Exp.

Shang, Y. and Huang, S. (2018) Multi-omics data driven investigations of metabolic diversity of plant triterpenoids. Plant $\mathrm{J}$.

Skelly, M.J., Frungillo, L. and Spoel, S.H. (2016) Transcriptional regulation by complex interplay between post-translational modifications. Curr Opin Plant Biol, 33, 126-132.

St-Pierre, P., Hassen, I.F., Thompson, D. and Perreault, J.P. (2009) Characterization of the siRNAs associated with peach latent mosaic viroid infection. Virology, 383, 178-182. 
Tolosa, J.M., Schjenken, J.E., Civiti, T.D., Clifton, V.L. and Smith, R. (2007) Column-based method to simultaneously extract DNA, RNA, and proteins from the same sample. Biotechniques, 43, 799-804.

Vorreiter, F., Richter, S., Peter, M., Baumann, S., von Bergen, M. and Tomm, J.M. (2016) Comparison and optimization of methods for the simultaneous extraction of DNA, RNA, proteins, and metabolites. Anal Biochem, 508, 25-33.

Wang, Y., Qu, J., Ji, S., Wallace, A.J., Wu, J., Li, Y., Gopalan, V. and Ding, B. (2016) A Land Plant-Specific Transcription Factor Directly Enhances Transcription of a Pathogenic Noncoding RNA Template by DNA-Dependent RNA Polymerase II. Plant Cell, 28, 10941107.

Wang, Y., Zirbel, C.L., Leontis, N.B. and Ding, B. (2018) RNA 3-dimensional structural motifs as a critical constraint of viroid RNA evolution. PLoS Pathog, 14, e1006801.

Wu, F.H., Shen, S.C., Lee, L.Y., Lee, S.H., Chan, M.T. and Lin, C.S. (2009) Tape-Arabidopsis Sandwich - a simpler Arabidopsis protoplast isolation method. Plant Methods, 5, 16.

Xiong, J., Yang, Q., Kang, J., Sun, Y., Zhang, T., Margaret, G. and Ding, W. (2011) Simultaneous isolation of DNA, RNA, and protein from Medicago truncatula L. Electrophoresis, 32, 321-330.

Zhang, H., Lang, Z. and Zhu, J.K. (2018) Dynamics and function of DNA methylation in plants. Nat Rev Mol Cell Biol, 19, 489-506.

Zheng, Y., Wang, Y., Ding, B. and Fei, Z. (2017) Comprehensive Transcriptome Analyses Reveal that Potato Spindle Tuber Viroid Triggers Genome-Wide Changes in Alternative Splicing, Inducible trans-Acting Activity of Phased Secondary Small Interfering RNAs, and Immune Responses. J Virol, 91. 


\section{Figure legends}

Figure 1. RT-PCR assessing RNA quality. RT-PCR cloning of a GFP fragment confirms the success purification of RNA.

Figure 2. Silver staining assessing the quality of enriched total proteins. (A) the comparison of proteins enriched from the flow-though (FL) using the GeneJET RNA purification kit with direct protein purification using RIPA buffer. (B) the comparison of proteins enriched from the RNA-depleted supernatant (Su) using the MagJET RNA purification kit with direct protein purification using RIPA buffer.

Figure 3. Immunoblotting detection of GFP. Immunoblotting successfully detected the GFP from our new protocol and from RIPA buffer purification. It is noticeable that our protocol enriched GFP from the flow-though of RNA purification column, supported by the better signals as compared with the RIPA purified samples.

Figure 4. Assessing the effect of DMSO. Two equal volume of RNA-depleted supernatant from MagJET RNA purification were treated with or without DMSO. Silver staining of SDS-PAGE gel (A) and immunoblotting (B) demonstrated that the supplementation of DMSO slightly enhanced the protein recovery rate, but may be omitted to simplify the protocol for high-throughput assays.

Figure 5. A flow chart demonstrating the procedures to co-purify DNA, RNA, and proteins. 
Figure 6. Co-purification of DNA, RNA, and proteins from protoplasts. PSTVd and 35S::GFP co-transfected Arabidopsis protoplasts were harvested two days posttransfection. DNA, RNA, and proteins were co-purified using our new method. (A) RNA gel blotting detected the replication of PSTVd in Arabidopsis protoplasts, and the ethidium bromide staining of ribosomal RNAs served as a loading control. The size illustration for RZ-Int RNA input is on the right panel. $\mathrm{HH}$ and $\mathrm{PC}$, hammerhead and paperclip ribozymes. (B) Immunoblotting detected the expressing of GFP, and histone H3 served as a loading control. (C) PCR using purified genomic DNA cloned a fragment from endogenous TFIIIA gene. c-PSTVd depicts circular PSTVd genome. 


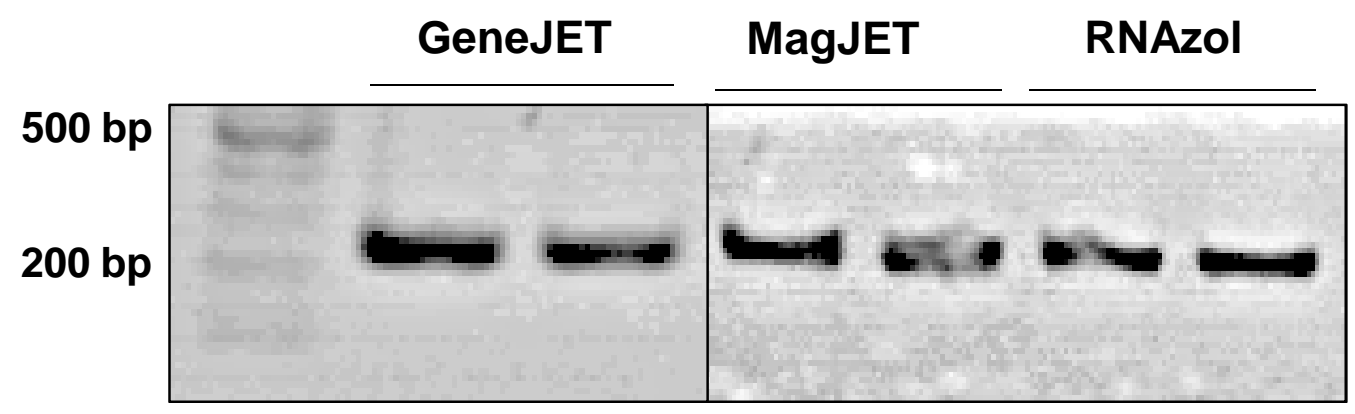

Figure 1. RT-PCR assessing RNA quality. RTPCR cloning of a GFP fragment confirms the success purification of RNA. 


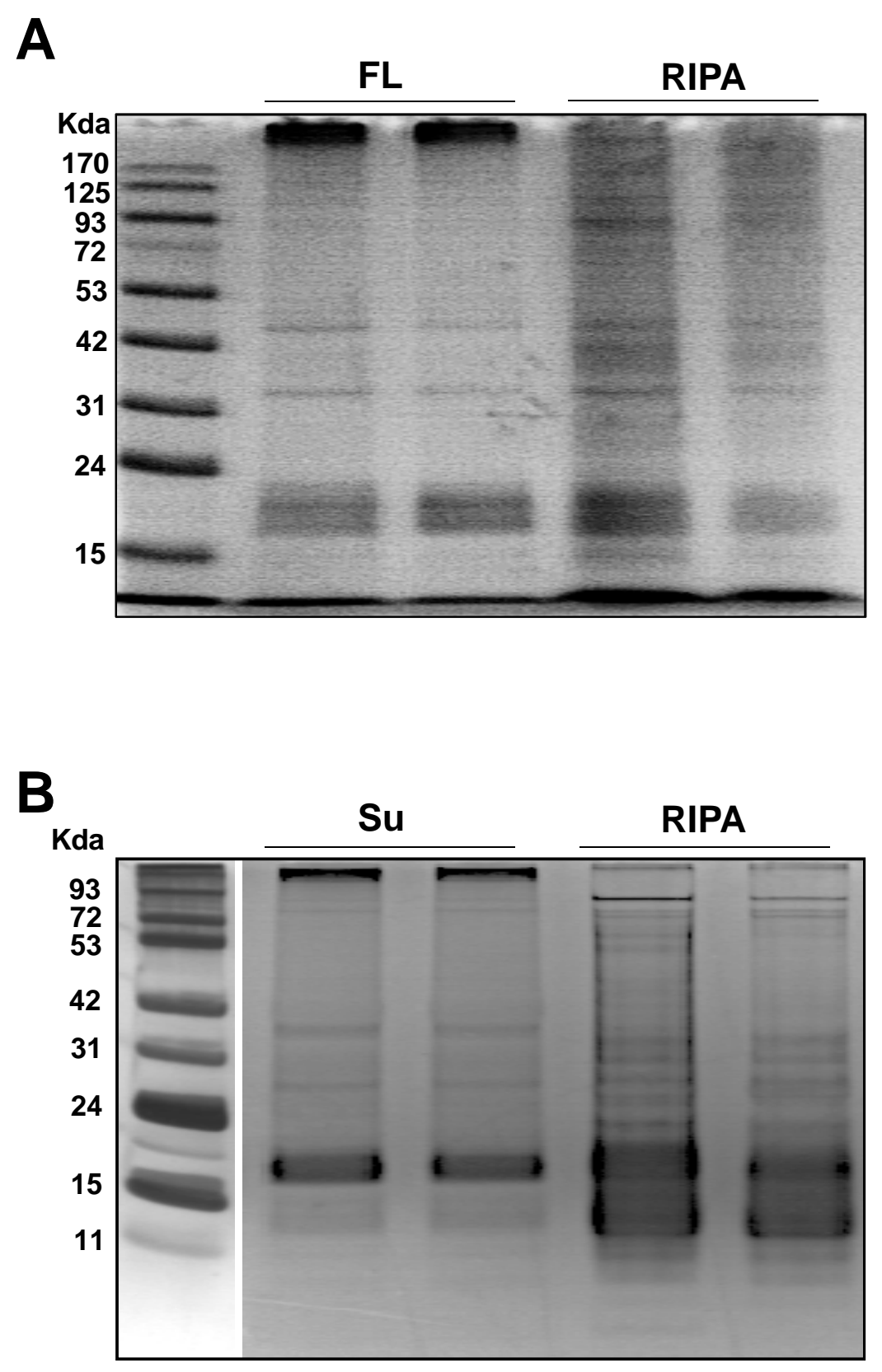

Figure 2. Silver staining assessing the quality of enriched total proteins. (A) the comparison of proteins enriched from the flow-though ( $F L$ ) using the GeneJET RNA purification kit with direct protein purification using RIPA buffer. (B) the comparison of proteins enriched from the RNA-depleted supernatant (Su) using the MagJET RNA purification kit with direct protein purification using RIPA buffer. 


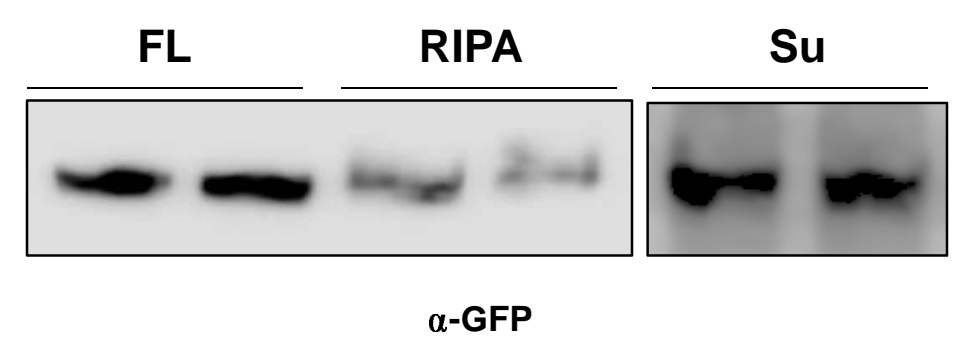

Figure 3. Immunoblotting detection of GFP. Immunoblotting successfully detected the GFP from our new protocol and from RIPA buffer purification. It is noticeable that our protocol enriched GFP from the flow-though of RNA purification column, supported by the better signals as compared with the RIPA purified samples. 


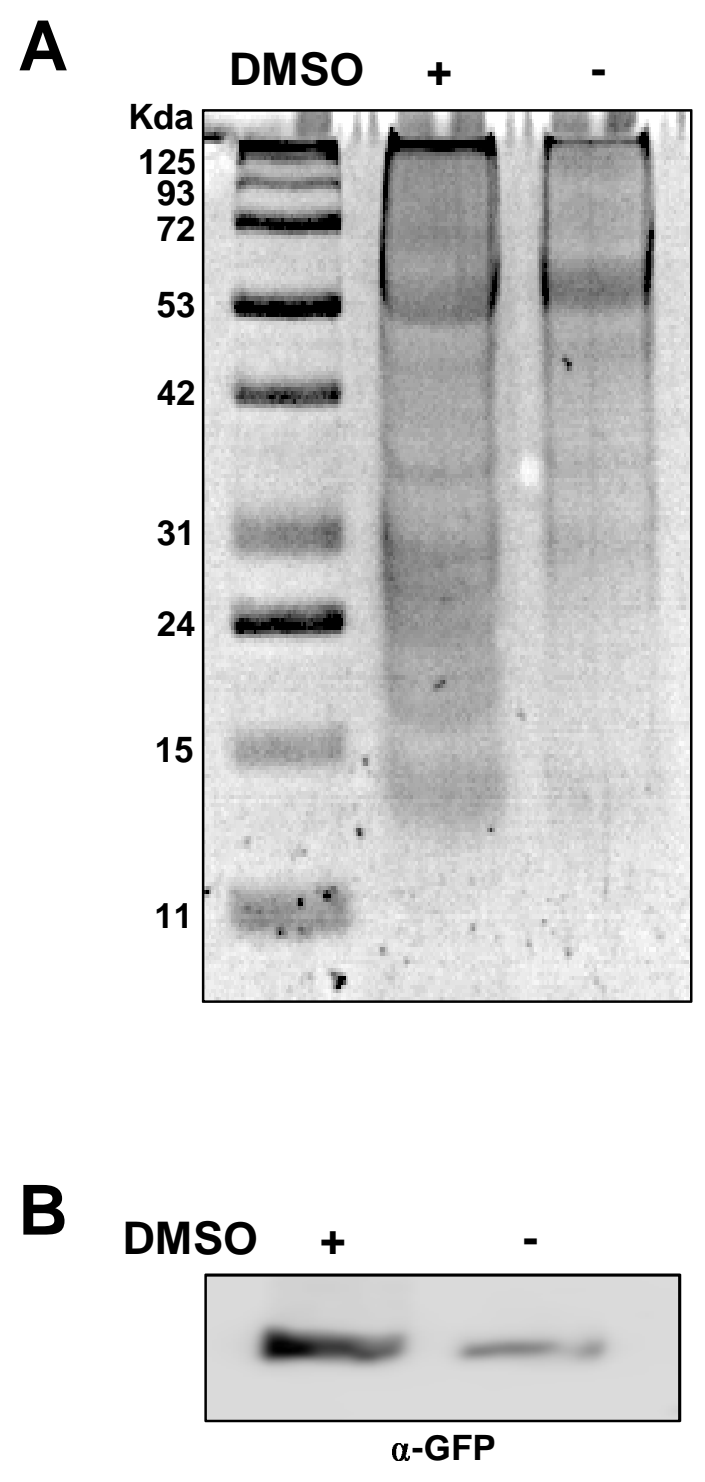

Figure 4. Assessing the effect of DMSO. Two equal volume of RNA-depleted supernatant from MagJET RNA purification were treated with or without DMSO. Silver staining of SDSPAGE gel (A) and immunoblotting (B) demonstrated that the supplementation of DMSO slightly enhanced the protein recovery rate, but may be omitted to simplify the protocol for high-throughput assays. 

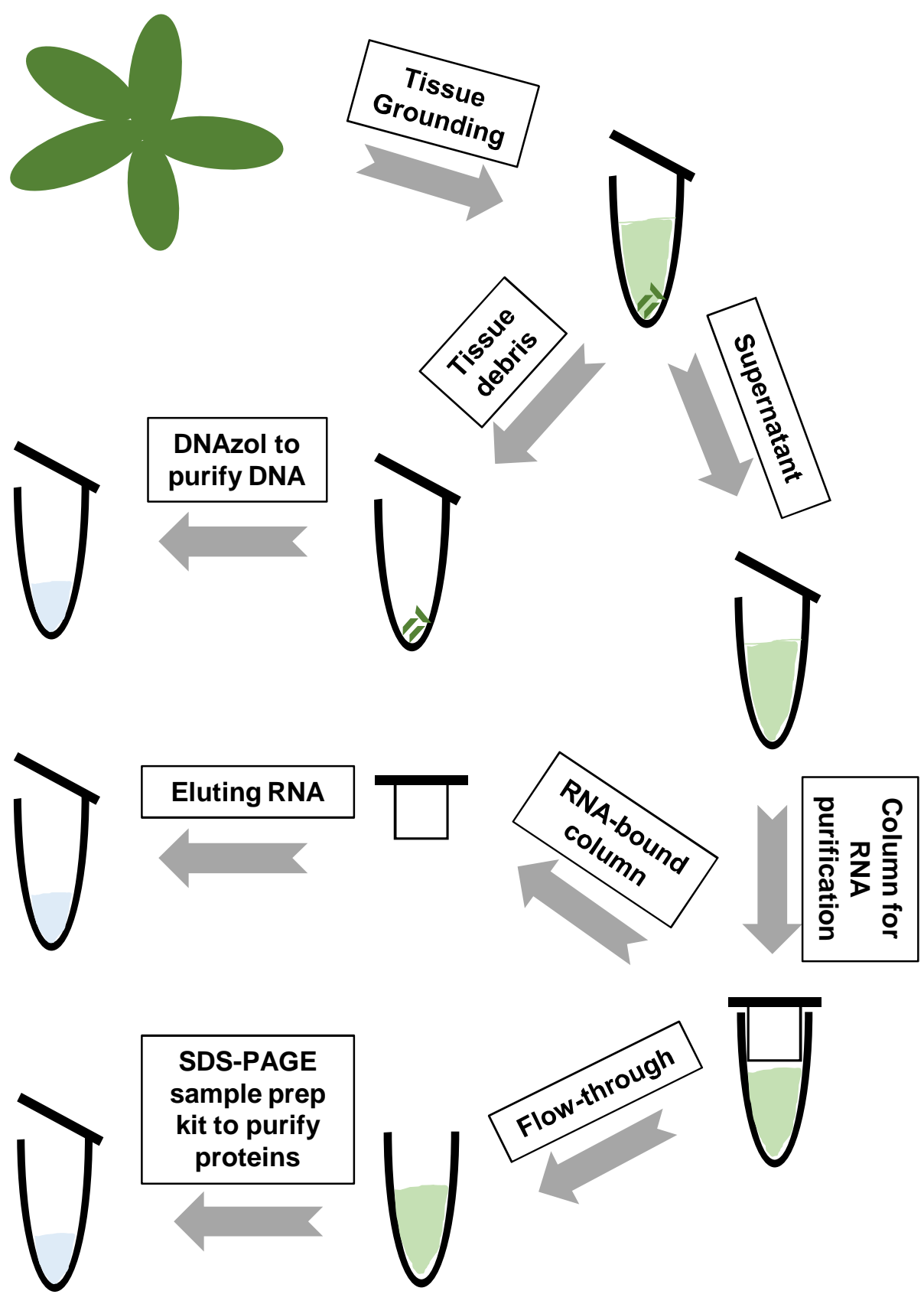

\section{Figure 5. A flow chart demonstrating the procedures to co-purify DNA, RNA and proteins.}




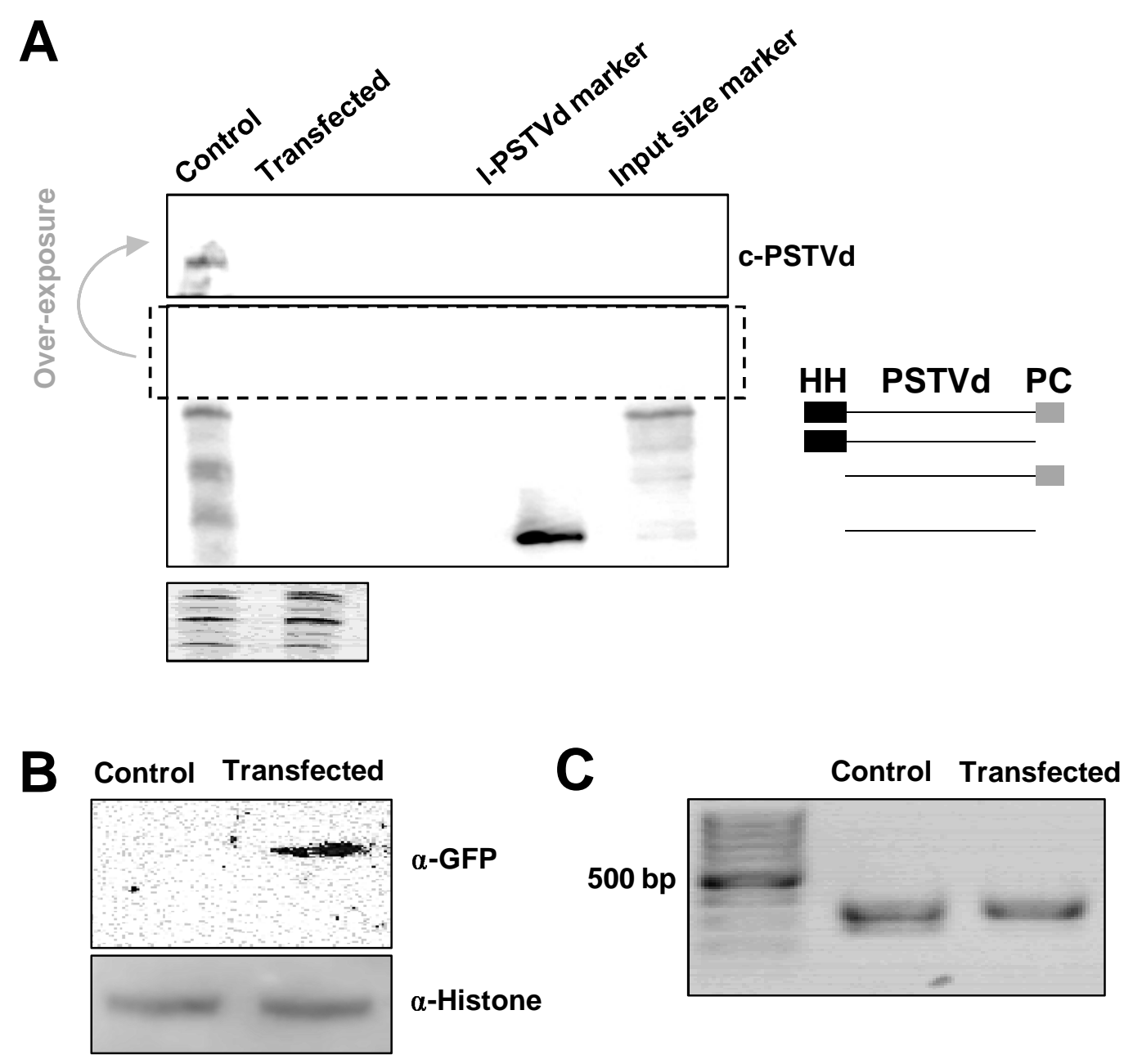

Figure 6. Co-purification of DNA, RNA and proteins from protoplasts. PSTVd and 35S::GFP co-transfected Arabidopsis protoplasts were harvested two days post-transfection. DNA, RNA and proteins were co-purified using our new method. (A) RNA gel blotting detected the replication of PSTVd in Arabidopsis protoplasts, and the ethidium bromide staining of ribosomal RNAs served as a loading control. The size illustration for RZ-Int RNA input is on the right panel. $\mathrm{HH}$ and $\mathrm{PC}$, hammerhead and paperclip ribozymes. (B) Immunoblotting detected the expressing of GFP, and histone $\mathrm{H} 3$ served as a loading control. (C) PCR using purified genomic DNA cloned a fragment from endogenous TFIIIA gene. cPSTVd depicts circular PSTVd genome. 